\title{
Advancing quantitative description of porosity in autogenous laser-welds of 304L stainless steel
}

\author{
Jonathan D Madison ${ }^{*}$, Larry K Aagesen², Victor WL Chan² and Katsuyo Thornton ${ }^{2}$
}

\author{
* Correspondence: \\ jdmadis@sandia.gov \\ ${ }^{1}$ Computational Materials \& Data \\ Science, Sandia National \\ Laboratories, 87185 Albuquerque, \\ NM, USA \\ Full list of author information is \\ available at the end of the article
}

\begin{abstract}
Porosity in linear autogenous laser welds of 304L stainless steel has been investigated using micro-computed tomography to reveal defect content in fifty-four welds made with varying delivered power, travel speed and focal lens. Trends associated with porosity size and frequencies are shown and interfacial measures are employed to provide quantitative descriptors of pore shape, directionality, interspacing and solid linear fraction. Lastly, the coefficient of variation associated with equivalent pore radii is reported toward a discussion of microstructural variability and the influence of process-parameters on such variability.
\end{abstract}

Keywords: Micro-computed tomography; Porosity; Stainless steel; Interfacial shape distribution; Interfacial normal distribution

\section{空

\section{Background}

Among joining and metal processing techniques used in industrial and scientific capacities, laser welding is relatively new. Due to its ability to supply high densities of power to very controlled areas with minimal peripheral excess heat input, it has become a rapidly growing and highly attractive joining process for metals [1,2]. Common interrogation practice for welds are often performed via post-mortem failure analysis [3], post-process radiography [4], or ultrasonic scan [5]. Typically, these evaluations provide an opportunity to identify the most probable cause of failure, or produce a qualitative understanding of the internal structure of the weld.

For most engineering metals, there exists a fairly clear inverse correlation between pore volume and mechanical properties such as strength or modulus with varying degrees of sensitivity. As a specific example, defects such as pores, occurring naturally or imposed artificially, have been shown to serve as preferred sites for the initiation or propagation of failure in creep in both conventional and high cycle fatigue of aluminum [6,7], a material system having high formability and broad applications. 304L stainless steel is unique in this regard as the effects of porosity on some material properties challenge intuition. Two examples in the literature which illustrate this phenomena can be found in the work of Boyce et al. [3] and Kuo and Jeng [8]. In the work of Boyce et al., autogenous continuous-wave and pulsed-wave laser welds were made across the gauge section of 304L stainless-steel tensile bars, which were subsequently strained to failure. While one weld schedule was noted to produce higher amounts of porosity than the other, no decrease in mechanical strength was observed. In the work 
of Kuo and Jeng, a variety of weld schedules were created for 304L stainless steel, where increasing porosity levels coincided with decreases in hardness and relatively small variations in yield strength. Additionally, the continuous-wave-laser weld sample, which contained higher amounts of porosity than any pulsed-wave-laser weld sample, demonstrated significantly higher tensile strength than all pulsed-wave-laser weld samples [8]. These findings suggest that the interplay of processing parameters may affect laser-welded microstructure in ways that complicate the individual effect of porosity, particularly in 304L. Furthermore, both examples illustrate that the effects of laserwelding induced porosity in 304L on certain mechanical properties is not clearly understood. We suggest that advancing the quantitative description of porosity in 304L laser weldments and relating them directly to carefully controlled weld parameters can assist in better understanding the concomitant effects of porosity in this ubiquitous and highly damage-tolerant material system.

Fortunately, for nearly all metallic systems, the parameters used to form the laserweld are among the most pivotal factors that determine the local microstructure. Typical processing parameters may include; shielding gas, laser power, power profile, filler material, travel speed and focal distance between the laser source and weld surface. The combination of these factors is often referred to as the 'weld schedule'. In this study, parameters of the weld schedule investigated have been limited to weld power, travel speed and focal length. Fortunately, recent advances in characterization and microstructure visualization have provided a rich set of tools being increasingly brought to bear on laser-weld induced porosity in a variety of metals [9-13]. The work presented here builds upon such investigations and utilizes micro-computed tomography and other emerging state-of-the-art three-dimensional (3D) characterization techniques to quantitatively relate porosity in autogenous laser-welds of 304L stainless-steel to specific processing parameters $[9,10,14]$.

\section{Methods}

Using a ROFIN-Sinar, Inc. CW $0.15 \mathrm{HQ}$, fiber-optic delivered Nd:YAG laser, over fifty unique weld-schedules were used to produce autogenous standing-edge seam welds in 304L stainless steel having an elemental composition of $\mathrm{Fe}-0.04 \mathrm{C}-18.12 \mathrm{Cr}-1.21 \mathrm{Mn}-$ $8.09 \mathrm{Ni}-0.028 \mathrm{~N}-0.022 \mathrm{P}-0.001 \mathrm{~S}-0.34 \mathrm{Si}$ (wt.\%). In all subsequent depictions, the $x$-direction denotes the weld width, the $z$-direction denotes the weld length and the $y$-direction denotes the weld depth, which is also the axis of incidence of the laser relative to the sample. For each sample, a standing-edge weld was formed by affixing two $2.54 \mathrm{~cm} \times$ $10.16 \mathrm{~cm} \times 0.1 \mathrm{~cm}$ flat plates together face-to-face by base-clamp, then laser-welding their upper seam at one of five constant travel speeds $\left(254 \mathrm{~mm} \times \mathrm{min}^{-1}, 508 \mathrm{~mm} \times \mathrm{min}^{-1}\right.$, $1016 \mathrm{~mm} \times \mathrm{min}^{-1}, 1524 \mathrm{~mm} \times \mathrm{min}^{-1}$ or $2032 \mathrm{~mm} \times \mathrm{min}^{-1}$ ) and at one of six delivered powers ranging from $200 \mathrm{~W}$ to $1200 \mathrm{~W}$ as measured with a Macken P2000Y laser power-probe. This parameter set included a total of 27 separate weld schedules and was performed for two separate focal lengths, $80 \mathrm{~mm}$ and $120 \mathrm{~mm}$, bringing the total weld schedule count to 54. For a complete matrix of the weld schedules investigated, see Table 1.

Following welding, micro-computed tomography $(\mu \mathrm{CT})$ was performed on each sample to reveal the size, location and morphology of the internal porosity. A Kevex PSX 10-65 W x-ray tube was employed using a $250 \mu \mathrm{A}$ current and $130 \mathrm{kV}$ operating 
Table 1 Maximum pore volume and total pores observed per case

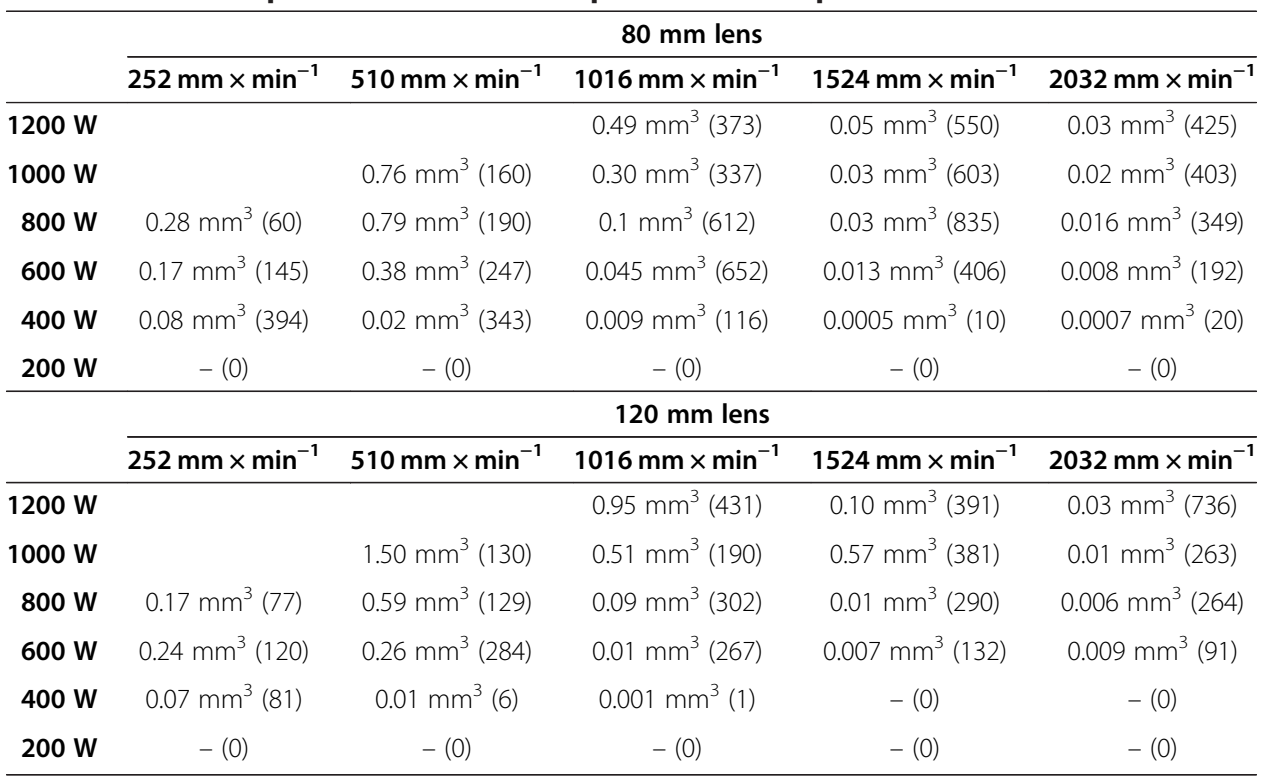

voltage. Samples were rotated for one full rotation at a speed of approximately $0.12^{\circ} / \mathrm{s}$. In an effort to identify optimal trade-offs in scanning time and resolution, multiple scanning resolutions ranging from 9-30 $\mu \mathrm{m}$ per voxel edge were employed with most scans being performed at $15 \mu \mathrm{m}$ per voxel edge, except the six weld schedules produced at $1200 \mathrm{~W}$. A minimum of ten contiguous voxels was imposed for identification as a pore across all datasets. To verify changes in tomographic resolution did not bias results, one weld produced at $1524 \mathrm{~mm} \times \mathrm{min}^{-1}$ was re-scanned at higher resolution. No significant differences in average pore size, maximum pore or total number of pores were observed. To provide a basis for comparison with more conventional methods, standard metallographic preparation and imaging via optical microscopy were performed on weld-sample cross-sections. Measurements of weld depth, width, crown height and pore volume fraction were also performed $[9,10]$. In these studies, pore volume fraction was shown to vary from $1-8 \%$ and result largely from key-hole collapse of the weld pool [13,15-18]. Transverse and longitudinal micrographs illustrating pore structures are shown in Figure 1 for three separate weld schedules made at $1200 \mathrm{~W}$.

Following tomography, three-dimensional reconstructions of pore populations were performed by two separate methods. A cone-beam reconstruction algorithm operating in tandem with the $\mu \mathrm{CT}$ experiment was carried out using VG StudioMax and twodimensional images derived from the $\mu \mathrm{CT}$ experiments were independently segmented in Adobe Photoshop ${ }^{\circ}$ and reconstructed using IDL ${ }^{\circ}$. This was done to transfer the data into a form amenable to higher-level morphological analyses such as interfacial and topological characterization, which will be discussed later. A series of images from IDL $^{\circ}$-generated reconstructions of weld porosity at $600 \mathrm{~W}$ and the previously identified five travel speeds are shown in Figure 2. The color designations for each pore set in Figure 2 have been utilized to denote a specific travel speed and will be employed throughout the paper to aid the reader in making associations between weld parameters in subsequent figures and plots. For two full color-coded matrices of the welds investigated, the reader may see the Additional files 1 and 2. 


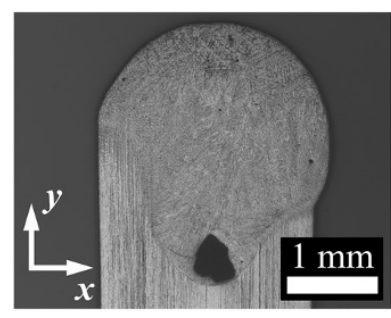

(a)

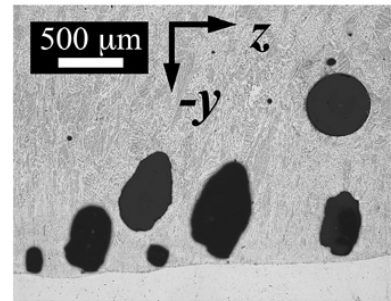

(d)

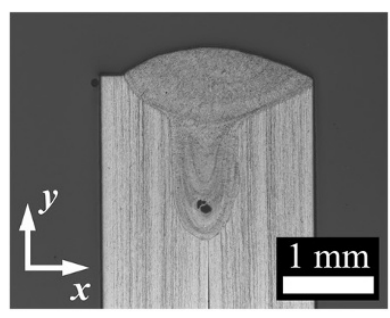

(b)

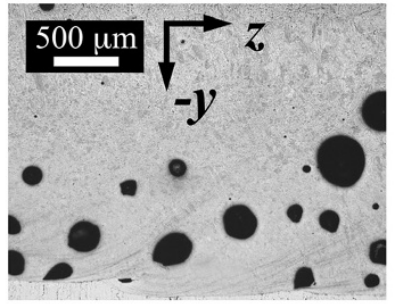

(e)

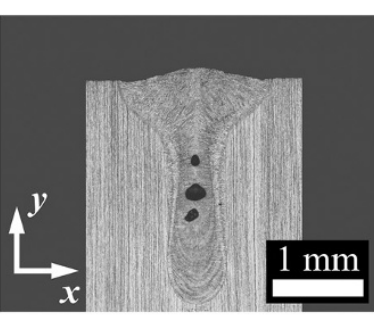

(c)

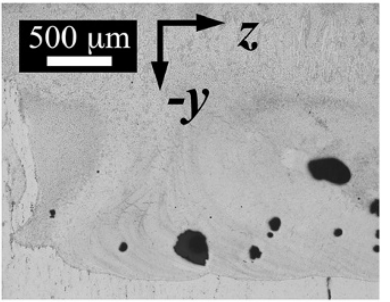

(f)

Figure 1 Transverse (a-c) and longitudinal (d-f) micrographs of welds produced under a power of $1200 \mathrm{~W}$ at (a, d) $1016 \mathrm{~mm} \times \mathrm{min}^{-1}$, (b, e) $1524 \mathrm{~mm} \times \mathrm{min}^{-1}$ and (c, e) $2032 \mathrm{~mm} \times \mathrm{min}^{-1}$. Transverse cross-sections show keyhole weld geometries including porosity and surrounding base metal, and longitudinal micrographs taken along welding direction further indicate scale of porosity present.

\section{Characterization}

\section{Pore characterization}

Utilizing the reconstructions obtained and the known voxel resolutions for each weld sample, physical measures of pore size, population and frequency were calculated for pores constituting ninety-percent or more of the voided space within each sample. These values serve as a baseline and comparison for readily employed measures of pore presence.

\section{Interfacial morphology}

Using tools developed by Voorhees et al. [19-24], the interfacial morphology of porosity was also examined. The primary tools used to illustrate these quantitative descriptors of shape are the interfacial shape [20,22] and interfacial normal [24] distributions. Given a triangulation representing the interface between two phases of a discretized microstructure, the mean $(H)$ and Gaussian $(K)$ curvatures can be calculated for each patch of interface according to the method of Meyer et al. [25]. These in turn, can be used to calculate the minimum and maximum principal curvatures, $\kappa_{1}, \kappa_{2}$ respectively, for each patch of interfacial area as follows.

$$
\begin{aligned}
& \kappa_{1}=H-\sqrt{H^{2}-K} \\
& \kappa_{2}=H+\sqrt{H^{2}-K}
\end{aligned}
$$

This analysis allows for categorization of all patches into a specific $\left(\kappa_{1}, \kappa_{2}\right)$ pairing. The method used to visualize this probability of principal curvature pairings is a twodimensional color contour plot in which the horizontal and vertical axes are $\kappa_{1}$ and $\kappa_{2}$, respectively, and the color indicates the probability associated with each pair of 


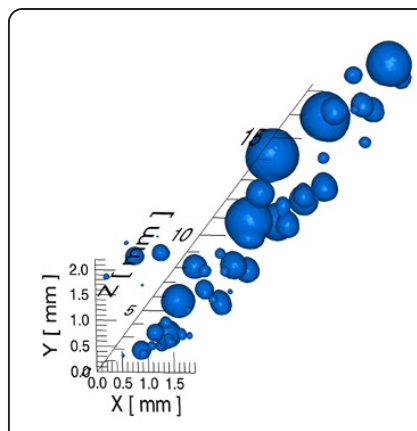

(a)

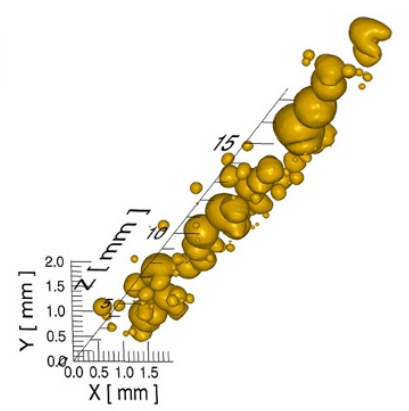

(b)

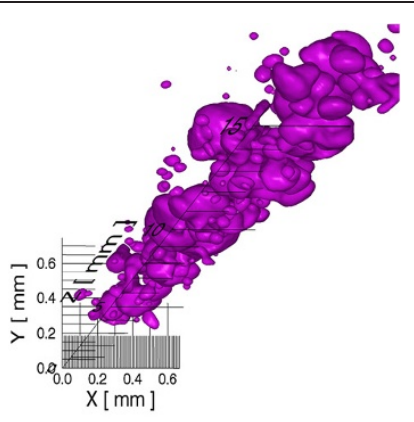

(c)

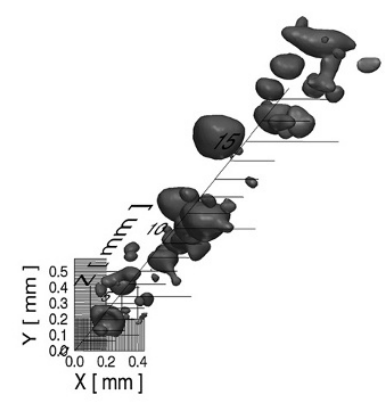

(d)

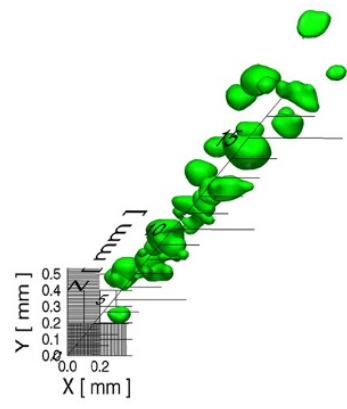

(e)

Figure 2 3D reconstructions of weld porosity produced at a focal length of $120 \mathrm{~mm}$ under a delivered power of $600 \mathrm{~W}$ and travel speeds of (a) $254 \mathrm{~mm} \times \mathrm{min}^{-1}$, (b) $508 \mathrm{~mm} \times \mathrm{min}^{-1}$, (c) $1016 \mathrm{~mm} \times \mathrm{min}^{-1}$, (d) $1524 \mathrm{~mm} \times \mathrm{min}^{-1}$, (e) $2032 \mathrm{~mm} \times \mathrm{min}^{-1}$, illustrating decreasing average pore size at higher travel speeds and highest shape complexity occurring at $1016 \mathrm{~mm} \times \mathrm{min}^{-1}$.

principal curvatures. This visualization technique is called the Interfacial Shape Distribution (ISD) $[20,22,23]$ and its descriptive legend is reproduced here for convenience, Figure 3. For the purposes of this study, the "L" phase, as illustrated in the figure, corresponds to the pores within welds (the figure was originally made for a solid-liquid system). To interpret the ISD, it is useful to arrange the curvature pairings into four major regions or categories. The uniqueness of these four categories is determined by the combination of positive or negative signs of $H$ and $K$. Patches in which both $H$ and $K$ are positive correspond to Region 1 , which are spherical or ellipsoidal with solid within. Patches in which the $H$ is positive and $K$ is negative correspond to region 2. Region 3 consists of patches that have negative $H$ and $K$ values. Regions 2 and 3 contain saddle shaped patches. Lastly, patches that have a negative $H$ and positive $K$ correspond to $\operatorname{Re}$ gion 4, which are spherical or ellipsoidal with the pore phase within. Based upon the population of each principal curvature within one complete three-dimensional reconstruction, which we will also refer to as a dataset, a probability can be assigned denoting the likelihood of encountering a particular pairing of principal curvatures for a given patch on the interface. It is important to note that the ISDs presented in this study have not been normalized by any characteristic length-scale and therefore illustrate a combined effect of both shape and size. As a result, the color bar associated with the ISDs presented later in the results section have units of $\mu \mathrm{m}^{2}$ so that the integration of the probability function over the entire ISD is equal to 1 . 


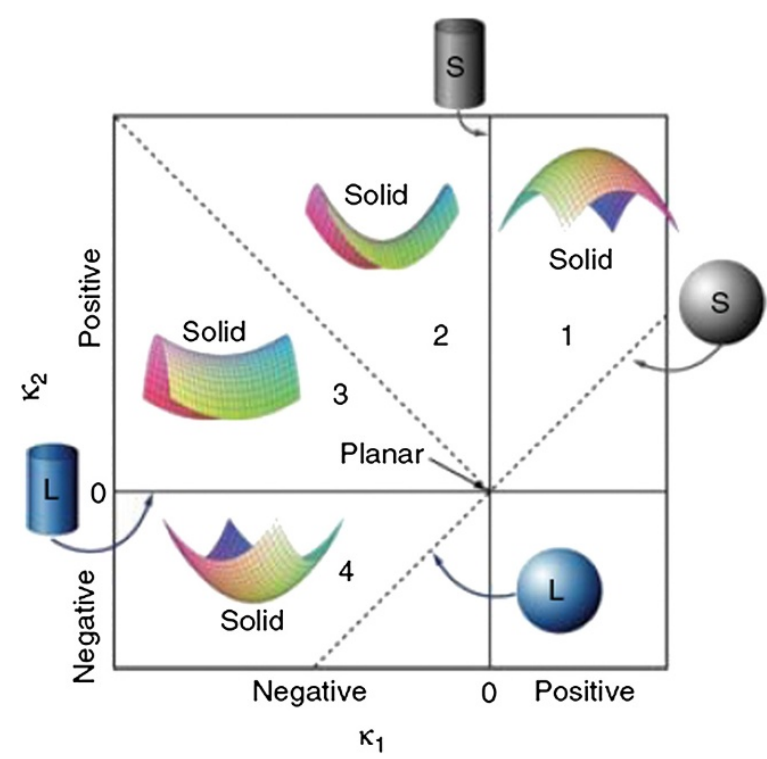

Figure 3 Interfacial Shape Distribution Legend indicating curvature types and their location within the ISD $[\mathbf{2 0}, \mathbf{2 2}$,23]. Reprinted from Acta Materialia, Vol. 54, issue 6, D. Kammer, P. Voorhees, "The Morphological Evolution of Dendritic Microstructures During Coarsening", pp. 1549-1558, (2006), with permission from Elsevier.

\section{Interfacial orientation}

The interfacial normal $(\hat{n})$ associated with each interfacial patch of a dataset is used to define a probability distribution for their orientation in three-dimensional space. The method used to visualize this probability distribution is the Interfacial Normal Distribution or IND [23,24]. In this visualization technique, the two-dimensional projection of a sphere with respect to a given axis displays the probability of occurrence of a given normal orientation. In this study, all INDs are presented as projections along the positive $z$-axis, which is also the direction of travel for the work-piece beneath the welding laser. Thus, the upper and lower hemispheres correspond to the direction toward and away from the laser, respectively. The color values at each location in the IND indicate the probability of encountering a particular normal based on the population of normals within the dataset. The color bar associated with each IND presented later in the section on results represents non-dimensional probability.

\section{Spatial analysis}

To better understand the spatial distribution of pore interfaces, we develop a method to calculate interfacial-distance distributions (IDD) that provide the probability distribution of the inter-pore distances, as measured between the pore surfaces nearest to one another. In this paper, we refer to the IDD of the pores as "pore-interspacing distribution" (PID). The method is based on the scheme for calculating the channel-size distributions of complex three-dimensional microstructures [26]. Further details regarding this methodology will be available in a forthcoming publication. Briefly, to calculate the interface-distance distribution, the image data of the 3D microstructure of the weld is first converted to a signed distance function, the magnitude of which represents the distance from the nearest pore-solid interface. The sign of the value indicates phase within the dataset. In this study, points inside the pores are represented as positive 
distances and points in the solid are represented as negative distances. Each isodistance of a microstructure is an isosurface defined by thresholding the distance function associated with the microstructure at a particular distance value. We refer to the regions with distance function values greater than the threshold as "voids" to distinguish them from the original pores as the sizes and topological characteristics of the voids differ from those of the pores. (Note that the interface of the microstructure is described by the isosurface with a threshold value of zero.) Topological changes in the isodistance structure from one threshold to another denote the feature sizes of the structure. The differences between isodistance structures resulting from thresholding in the positive and negative directions are illustrated in the two-dimensional schematics of Figure 4, where the value of the distance function is represented by grayscale and the isocontour (analogous to isosurface in three dimensions) of each threshold value is marked by a black line.

When the isodistance structures join together from thresholding with a negative threshold value, a change in the number of voids arises. This occurs at a distance value corresponding to half of the pore interspacing (the distance between interfaces at the narrowest point). Since we are examining systems that contain a variety of spatial distributions of pores, PIDs are calculated by measuring the rate at which pores are joining as a function of the distance threshold. Specifically, the PID is calculated by taking the negative derivative ( -1 times the derivative) of the number of voids as a function of twice the distance threshold. Numerically, a central differencing method is used to calculate the derivatives. Each point in the PID represents the probability of finding a pair of pores with the pore interspacing at the corresponding distance threshold value.

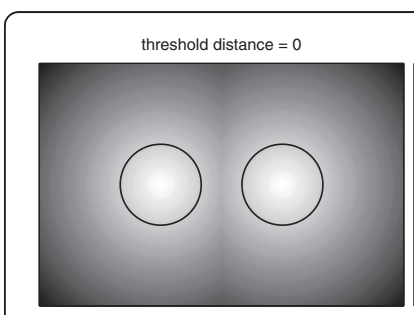

(a)

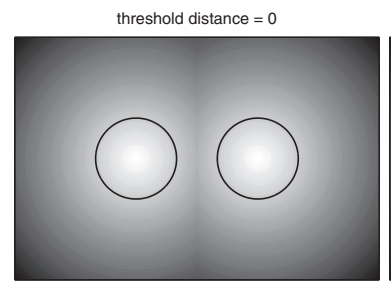

(d)

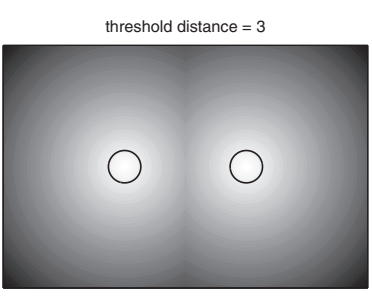

(b)

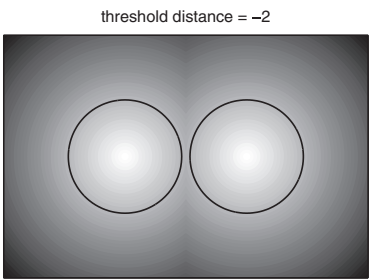

(e)

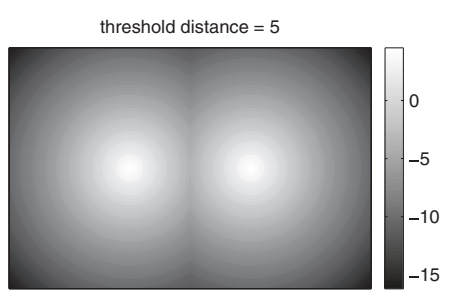

(c)

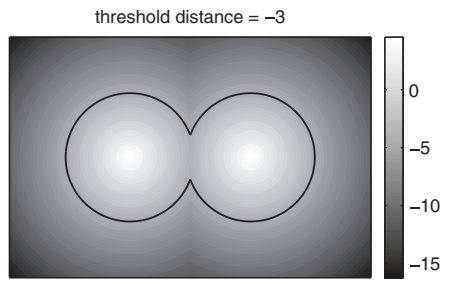

(f)

Figure 4 Two-dimensional schematic of the isodistance structures of two circular pores embedded in a uniform matrix where grayscale represents the values of the distance function. The interfaces of the two pores are denoted by the black lines at a threshold value of zero, (a) and (d). Isocontour structures (isodistance structures in three dimensions) become smaller with increasingly positive threshold values (b) and eventually disappear when the threshold value exceeds the radius of the pore (c). Isocontour structures become larger with increasingly negative threshold values (e) and eventually join together when the magnitude of the threshold value exceeds one half of the nearest distance between the two pores (f), causing a change in the number of voids. 
Furthermore, a characteristic pore interspacing is calculated by taking the weighted mean of the pore interspacing.

Similarly, when the isodistance structures are created for positive threshold values, a change in the number of voids also arises when the threshold becomes larger than the largest distance function value within a pore. The corresponding distance value is half the smallest dimension (the radius for a sphere) of the pore. Once again, measuring the rate at which pores disappear as a function of the distance threshold gives a pore-size distribution. Lastly, the characteristic pore interspacing is scaled to yield solid linear fraction (SLF):

$$
S L F=\frac{R}{r+R}
$$

where $R$ is one half of the characteristic pore interspacing and $r$ is the characteristic pore radius. The SLF provides a measure of local linear fraction of solid along the path connecting the center of the particles and passing through the narrowest matrix region. Unlike pore volume fraction, another commonly used measure of density, the SLF does not depend on the volume used for the calculation. This is of particular note for each weld schedule studied here, as laser weld porosity is generally a localized phenomena often occurring at the centerline of the weld and not distributed homogeneously throughout the weld. Furthermore, the SLF is useful as it yields a quantitative metric of solid material between regions of densely populated pores relative to the size of pores present. It is expected that this type of spacing sensitivity metric would have a strong influence on the mechanical properties of the weld.

\section{Results and discussion}

Population statistics

As mentioned previously, a variety of population metrics were extracted from each weld schedule including but not limited to average and maximum pore volume, linear frequency, and total number of pores observed per weld case. Average pore volume for both focal lengths are shown in Figure 5 where each data series corresponds to a specific travel speed and data are presented as a function of delivered power. The trend is consistent in that for a given travel speed, increases in welding power produce larger pores. Slower travel speeds also generally produce larger pores with some exceptions to this trend. Linear frequency was obtained by taking the total number of observed pores for a given weld and dividing it by the weld length. Figure 6 illustrates a more complex dependence of linear frequency on power and travel speed. With the exception of the $2032 \mathrm{~mm} \times \mathrm{min}^{-1}$ data series, an inflection point for the maximum frequency of pores was observed across all series of travel speeds, indicating that fewer pores can be obtained by increasing power delivered while maintaining the same travel speed. Furthermore, increases in travel speed shift the inflection point for diminishing pore presence to higher powers. An inflection point may be observed for the $2032 \mathrm{~mm} \times \mathrm{min}^{-1}$ travel speed at higher powers as the data suggests an inflection point for this travel speed may lie just beyond the bounds of this study. While the aforementioned trends are consistent across both focal lenses, it is interesting to note that the $120 \mathrm{~mm}$ lens produces higher average porosity volume (Figure 5) but generally lower frequencies (Figure 6) at all parameter sets. In Table 1, maximum pore volume observed and total pore 


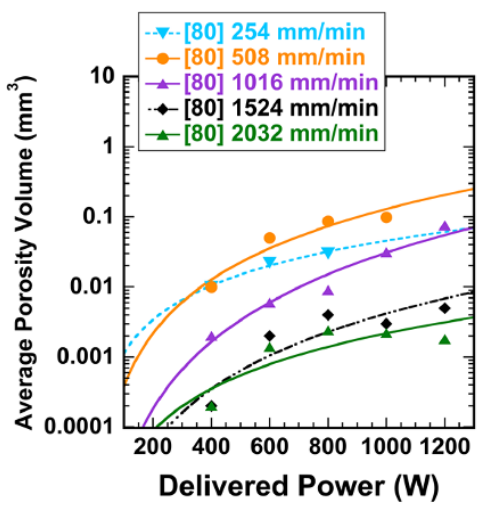

(a)

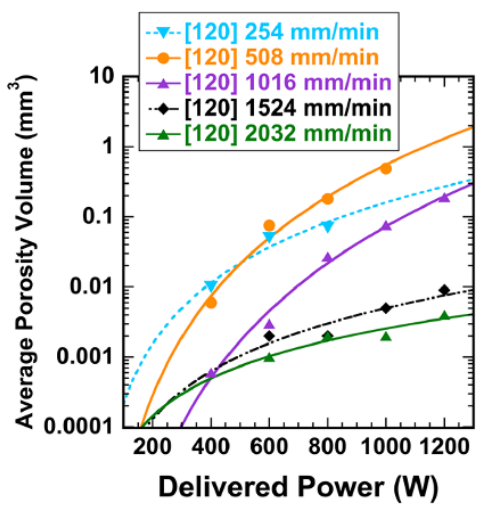

(b)

Figure 5 Average pore volume as a function of weld power under (a) $80 \mathrm{~mm}$ focal lens and (b) $120 \mathrm{~mm}$ focal lens showing an increase in pore volume with weld power and decreases in travel speed.

populations within each weld schedule are shown, with population counts denoted within parentheses. No porosity was observed in any weld produced at $200 \mathrm{~W}$ nor at higher travel speeds at $400 \mathrm{~W}$ under the $120 \mathrm{~mm}$ focal length.

\section{Interfacial shape distributions}

Interfacial shape distributions for porosity observed at $600 \mathrm{~W}$ across all five travel speeds are shown for a focal length of $120 \mathrm{~mm}$ in Figure 7. All ISDs are plotted on a uniform color scale in units of square microns to aid in comparison. In the discussion in this section and those following, welds are grouped by travel speed into three categories: low (254 and $508 \mathrm{~mm} \times \mathrm{min}^{-1}$ ), moderate $\left(1016 \mathrm{~mm} \times \mathrm{min}^{-1}\right.$ only), and high (1524 and $2032 \mathrm{~mm} \times \mathrm{min}^{-1}$ ). At low travel speeds, the majority of interfacial patches lie in Region 4 of the ISD, which largely corresponds to elliptical shapes with the edge of the shape distributions reaching the $\kappa_{1}=\kappa_{2}$ line, which corresponds to completely

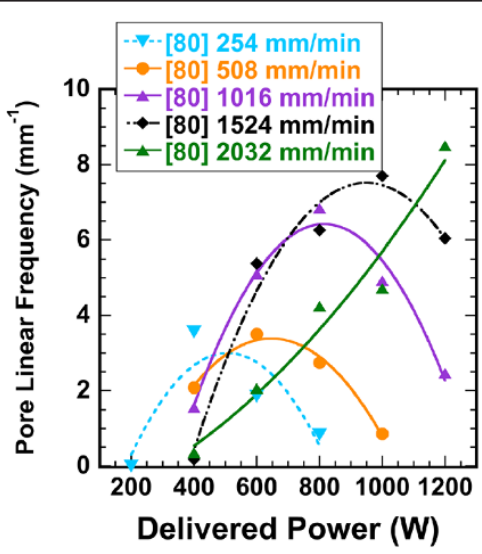

(a)

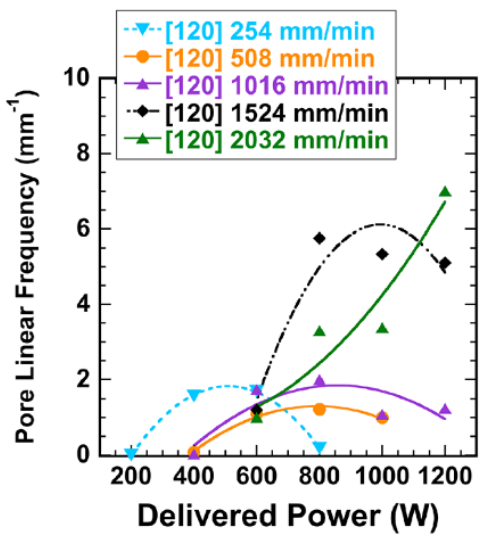

(b)

Figure 6 Pore frequency per unit length as a function of weld power under (a) $80 \mathrm{~mm}$ focal lens and (b) $120 \mathrm{~mm}$ focal lens. 


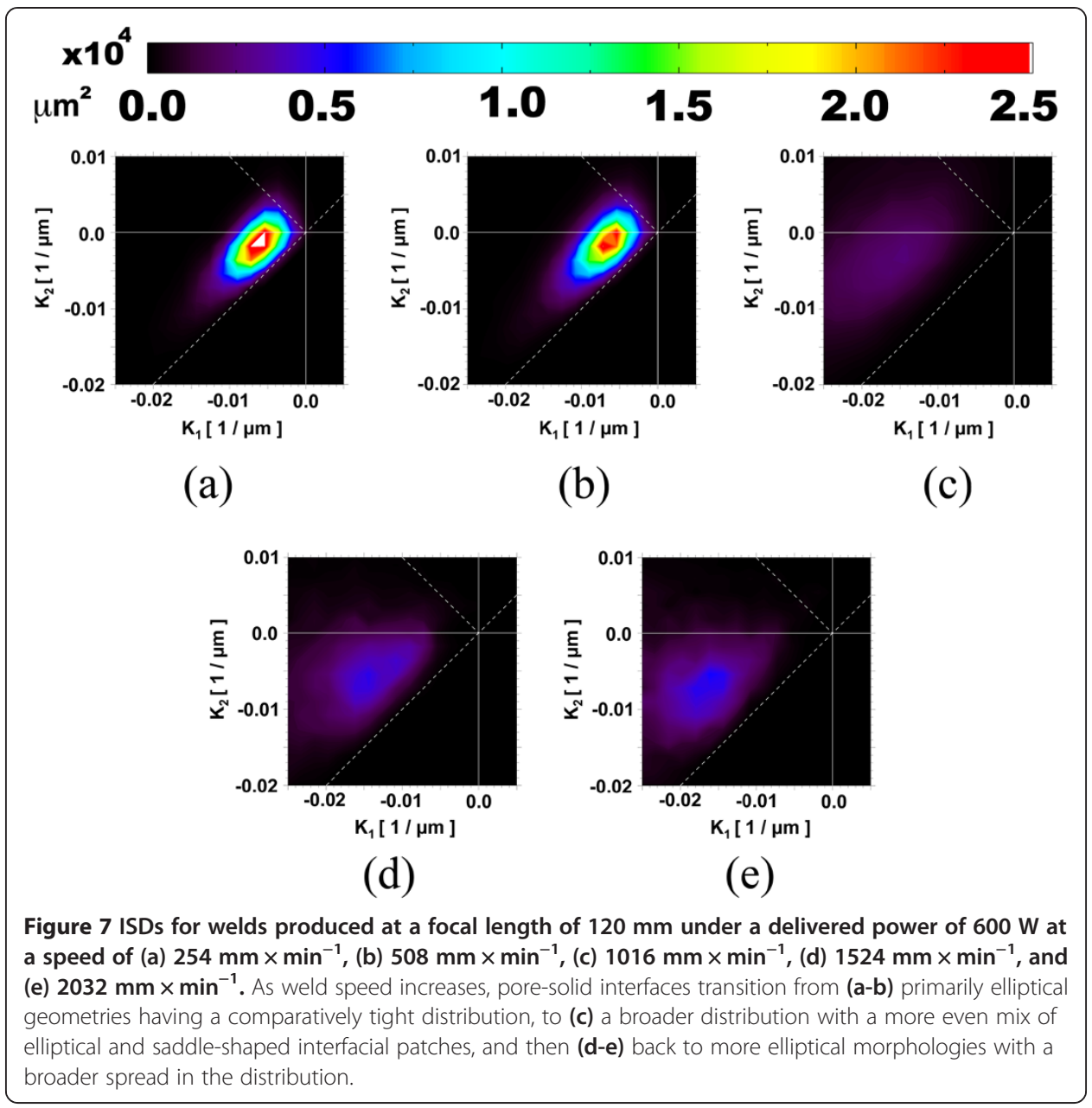

spherical shapes. For low travel speeds, the shape distributions also extend into Region 3 due to the presence of some saddle-shaped patches, as can be seen in Figure $2 \mathrm{~b}$ in the "kidney bean"-shaped void at the upper right corner of the 3D reconstruction. At moderate travel speed, curvature pair maxima tend to lie upon the $\kappa_{1}=0$ axis with a broad spread into regions 3 and 4 . At this moderate speed, the distribution does not approach the $\kappa_{1}=\kappa_{2}$ line closely, providing indication that voids are less spherical and generally more complex in nature. At high travel speeds, shape distributions are more diffuse than at low speeds and the overwhelming majority of the curvature distributions reside in Region 4. Additionally, the peaks of these shape distributions are again very close to the $\kappa_{1}=\kappa_{2}$ line, indicating voids are rather spherical at these higher travel speeds with very few saddle-shaped patches. The ISD peaks shift toward lower magnitudes of curvatures with increasing travel speed as observed in Figure 7, which is consistent with the fact that average pore volume decreases at higher travel speeds as shown in Figure 5.

The curvature distributions for a given travel speed are rather consistent across all power levels. The primary difference in ISDs relating to power variation, see Additional file 3,4 is that the peak of the curvature distributions exist at increasingly negative values of $\kappa_{2}$ with decreases in power. This change corresponds to more spherical pore morphologies being formed with decreases in weld power. These trends were observed 
consistently across both focal length welds. A full set of calculated ISDs for all weld cases in this study having more than twenty pores each are included in the Additional file 3,4 to this article.

\section{Interfacial normal distributions}

Interfacial normal distributions with respect to the positive $z$-axis are shown in Figure 8 for porosity in weld samples discussed in the previous section, again at $600 \mathrm{~W}$ and under a focal length of $120 \mathrm{~mm}$. Each IND is plotted with a uniform color scale with a maximum of $3.0 \times 10^{-3}$ (units of dimensionless probability) to enable direct comparison. At moderate and high travel speeds, local anisotropy in directionality appears such that pronounced clusters of normal orientations populate the lower and upper hemispheres of each projection in Figure $8 \mathrm{c}-\mathrm{e}$. Taken together with the ISDs, the net change in pore morphology with travel speed can be summarized as follows. At low travel speeds, pores are on average larger and are mainly near spherical or ellipsoidal (Figure $7 \mathrm{a}-\mathrm{b}$ ). At the same time, the orientation of the normals are more isotropic, as observed from the more uniform INDs (Figure $8 \mathrm{a}-\mathrm{b}$ ). Thus, we can conclude that ellipsoidal pores are randomly oriented. At the moderate travel speed, pore sizes decrease, and pore morphologies become less ellipsoidal and more irregular (Figure 7c), and the IND shows preferential normal orientation toward and away from the incident laser (see the enhanced probability in the upper and lower hemispheres in Figure 8c). At high travel speeds, pore morphologies once again become closer to spherical, with some ellipticity still present, as evidenced by the fact that the peaks of the ISDs do not lie directly on the $\kappa_{1}=\kappa_{2}$ lines (Figure $8 \mathrm{~d}-\mathrm{e}$ ). Also, as in the moderate-speed case, interfacial normals are aligned with the direction of laser incidence, resulting in the

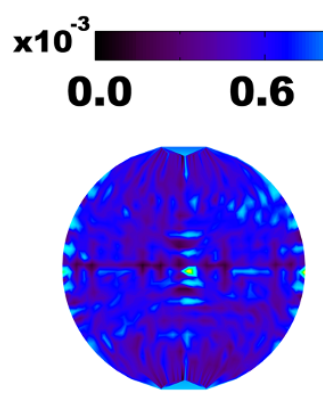

(a)

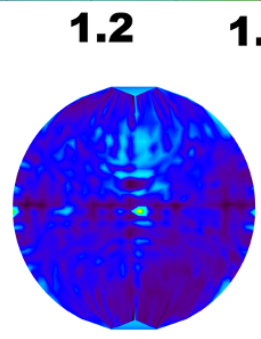

(b)
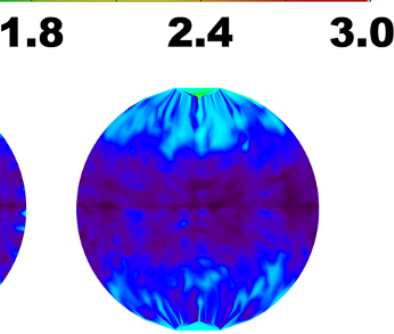

(c)

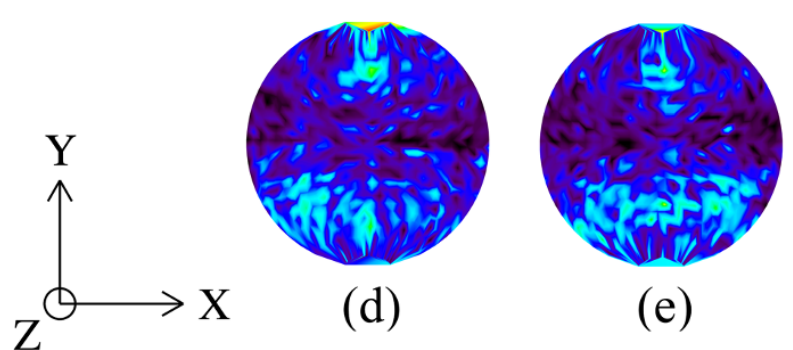

Figure 8 INDs for weld porosity produced at a focal length of $120 \mathrm{~mm}$ under a delivered power of $600 \mathrm{~W}$ and (a) $254 \mathrm{~mm} \times \mathrm{min}^{-1}$, (b) $508 \mathrm{~mm} \times \mathrm{min}^{-1}$, (c) $1016 \mathrm{~mm} \times \mathrm{min}^{-1}$, (d) $1524 \mathrm{~mm} \times \mathrm{min}^{-1}$, (e) $2032 \mathrm{~mm} \times \mathrm{min}^{-1}$. At low travel speeds, the orientation of pore-solid interfaces is nearly isotropic. As travel speed increases (a to e), the increased probabilities in the upper and lower hemispheres show that interfaces increasingly align with the $y$-axis, which is the axis of incidence of the laser. 
peaks of the INDs occurring in the upper and lower hemispheres at these high speeds. The same trends were observed in the negative $z$-axis projections as well. Succinctly stated, increasing weld speed results in decreased pore sizes and in interfacial normals preferentially orienting in the direction of laser incidence (both toward and away from). These trends in ISDs and INDs were observed across all powers and across both focal lengths. For completeness, calculated INDs for all weld cases in this study are also included in the Additional file 5, 6, 7 and 8 to more fully demonstrate the trend.

\section{Pore interspacing}

The pore-interspacing and pore-size distributions were calculated for a total of 18 weld schedules to more closely examine the interspacing of pores in three dimensions as a function of weld power and weld speed. These cases span both focal lenses and include welds for which power was varied while travel speed was held constant at $1016 \mathrm{~mm} \times$ $\min ^{-1}$ and for which the travel speed was varied for a constant power of $600 \mathrm{~W}$; see Table 2. These selections traverse the central column and row of both sample matrices where porosity was observed, shown in Table 1, encompassing median values for mmscale laser-welds. The probability distributions in Figures 9 and 10 were calculated as described in the section on spatial analysis. To alleviate redundancy, PIDs for only the $120 \mathrm{~mm}$ focal length welds are plotted in Figure 9, as the trends illustrated here are consistent for the $80 \mathrm{~mm}$ focal length results as well. In Figure 9, each data series represents a speed of $1016 \mathrm{~mm} \times \mathrm{min}^{-1}$ under a different power. Overall, the poreinterspacing probability distributions shift to higher values of distances as the weld power increases. Stated simply, pores are spaced farther apart at higher power.

Pore interspacing was also calculated for various weld speeds, as shown in Figure 10. Again, to reduce redundancy and to make the trend clear, only select results are shown for welds made at multiple speeds in conjunction with the $120 \mathrm{~mm}$ focal length at $600 \mathrm{~W}$. While the probability of finding pores at interspacing distances below $250 \mathrm{mi}$ crons is relatively high across all cases, the distributions appear to be broader for low and high travel speeds, with the high travel-speed case potentially exhibiting a bimodal

Table 2 Pore interspacing, radius and SLF as functions of weld power and speed

\begin{tabular}{|c|c|c|c|c|c|c|}
\hline \multirow[b]{2}{*}{$\begin{array}{l}\text { Weld power } \\
\text { (W) }\end{array}$} & \multicolumn{3}{|c|}{$80 \mathrm{~mm}$ lens } & \multicolumn{3}{|c|}{$120 \mathrm{~mm}$ lens } \\
\hline & $\begin{array}{l}\text { Pore interspacing } \\
(\mu \mathrm{m})\end{array}$ & $\begin{array}{l}\text { Pore radius } \\
\qquad(\mu \mathrm{m})\end{array}$ & SLF & $\begin{array}{l}\text { Pore interspacing } \\
(\mu \mathrm{m})\end{array}$ & $\begin{array}{l}\text { Pore radius } \\
\qquad(\mu \mathrm{m})\end{array}$ & SLF \\
\hline 400 & $300(14.8)$ & $51(7.4)$ & $0.75(0.05)$ & - & - & - \\
\hline 600 & $124(9.1)$ & $52(4.6)$ & $0.54(0.05)$ & $78(9)$ & $41(4.5)$ & $0.49(0.07)$ \\
\hline 800 & 144 (9.2) & $69(4.6)$ & $0.51(0.04)$ & $107(9)$ & $88(4.5)$ & $0.38(0.04)$ \\
\hline 1000 & $170(14.2)$ & $82(7.1)$ & $0.51(0.05)$ & $110(14.6)$ & $104(7.8)$ & $0.35(0.05)$ \\
\hline 1200 & 240 (15.5) & $89(7.8)$ & $0.58(0.05)$ & $210(20)$ & $120(10)$ & $0.47(0.05)$ \\
\hline $\begin{array}{c}\text { Weld speed } \\
\left(\mathrm{mm} \times \mathrm{min}^{-1}\right)\end{array}$ & $\begin{array}{l}\text { Pore interspacing } \\
(\mu \mathrm{m})\end{array}$ & $\begin{array}{l}\text { Pore radius } \\
\qquad(\mu \mathrm{m})\end{array}$ & SLF & $\begin{array}{l}\text { Pore interspacing } \\
(\mu \mathrm{m})\end{array}$ & $\begin{array}{l}\text { Pore radius } \\
\quad(\mu \mathrm{m})\end{array}$ & SLF \\
\hline 252 & $270(14.8)$ & $108(7.4)$ & $0.55(0.04)$ & 340 (14.6) & $129(7.8)$ & $0.57(0.04)$ \\
\hline 510 & $170(14.8)$ & $142(7.4)$ & $0.37(0.04)$ & $160(14.6)$ & $124(7.8)$ & $0.40(0.04)$ \\
\hline 1016 & $124(9.1)$ & $52(4.5)$ & $0.54(0.05)$ & $78(9)$ & $41(4.5)$ & $0.49(0.07)$ \\
\hline 1524 & $110(14.3)$ & $63(7.1)$ & $0.47(0.07)$ & 230 (14.6) & $51(7.8)$ & $0.70(0.06)$ \\
\hline 2032 & $190(14.3)$ & $57(7.1)$ & $0.62(0.06)$ & 310 (14.6) & $58(7.8)$ & $0.72(0.05)$ \\
\hline
\end{tabular}

Variable weld powers shown are for a constant travel speed of $1016 \mathrm{~mm} \times \mathrm{min}^{-1}$ and variable weld speeds shown are for a constant power of $600 \mathrm{~W}$. 


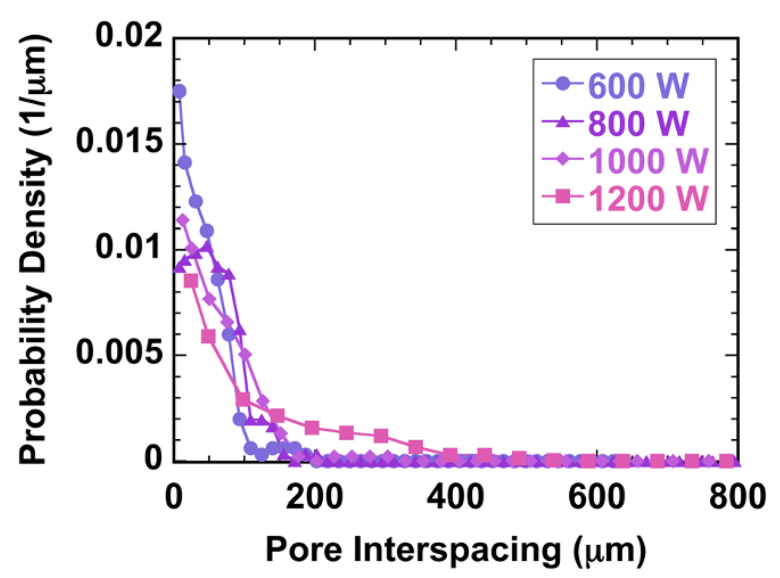

Figure 9 Pore-interspacing distributions with variation in power for $120 \mathrm{~mm}$ focal length weld series. The probability distribution shifts to higher values of distances as weld power is increased.

distribution. However, the statistics are insufficient to conclusively determine whether a bimodal distribution exists; further examination of larger weld samples or a larger number of samples under the same processing parameters are required to do so.

The characteristic pore interspacing, characteristic pore radius, and SLF are listed in Table 2 for all pore structures considered in Figures 9 and 10. To better illustrate the variation with process parameters, calculated SLF are plotted as functions of delivered power and weld speed across all process pairs examined in Figure 11. The method for calculating pore interspacing and radius is accurate up to half a voxel [26]. Therefore, the accuracy of each calculation is dependent on the resolution of the measurements; the uncertainty is listed within parenthesis in Table 2 and shown by error bars in Figure 11.

As described earlier, pore interspacing is a measure of the proximity of pores in the weld structure, while the SLF measures the proximity of pores relative to the distance between their centers and the characteristic pore size. For the samples where weld power is varied, the smallest pore interspacing was found at $600 \mathrm{~W}$ for a speed of

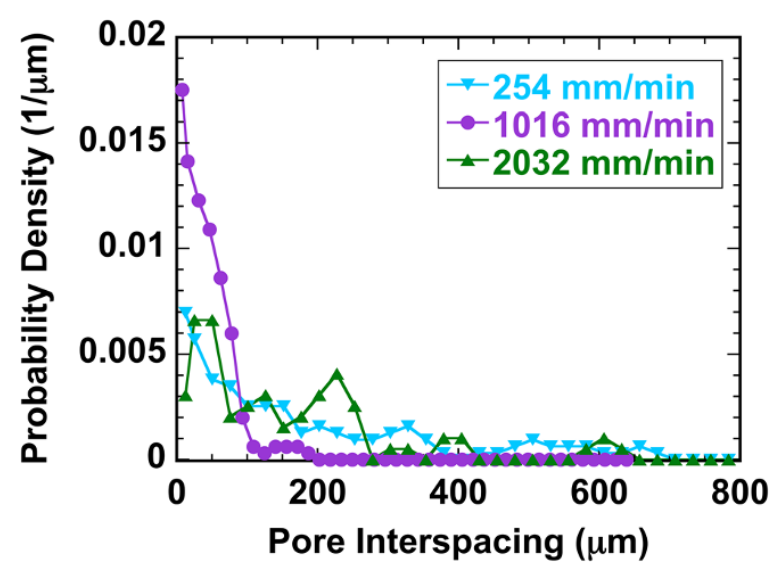

Figure $10 \mathrm{~A}$ comparison of pore-interspacing distributions with varying weld speed for $120 \mathrm{~mm}$ focal length at $600 \mathrm{~W}$. At the high $\left(2032 \mathrm{~mm} \times \mathrm{min}^{-1}\right)$ and low $\left(254 \mathrm{~mm} \times \mathrm{min}^{-1}\right)$ speeds, several pores are found at large distances from one another, while at the medium $\left(1016 \mathrm{~mm} \times \mathrm{min}^{-1}\right)$, weld speed pores are clustered relatively closely. 


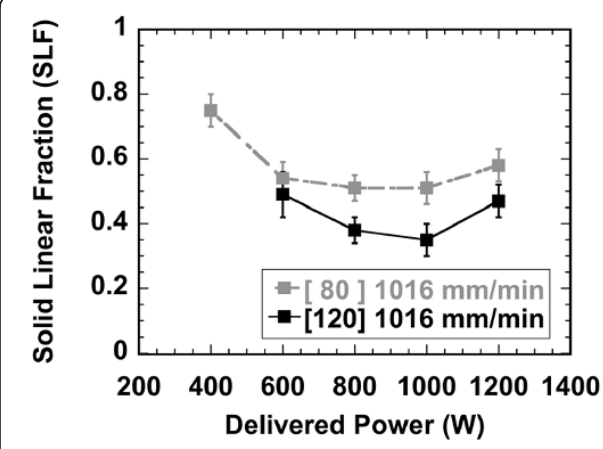

(a)

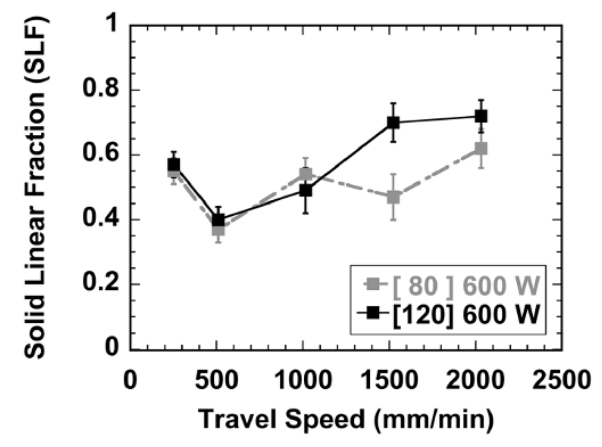

(b)

Figure $11 \mathrm{SLF}$ as a function of weld (a) power and (b) speed. With respect to variations in travel speed, minimum SLF was observed to occur at $510 \mathrm{~mm} \times \mathrm{min}^{-1}$. With respect to variations in power, SLF was lowest in the range of 800-1000 W, which is noteworthy as this range of powers coincides with the parameters that produce high pore frequencies per unit length across all weld schedules investigated (see Figure 6). The error bars show the calculation uncertainty presented in parenthesis in Table 2.

$1016 \mathrm{~mm} \times \mathrm{min}^{-1}$ for both 80 and $120 \mathrm{~mm}$ lens welds (see Table 2), while the minimum SLF occurs at weld powers of $800-1000 \mathrm{~W}$ for the same travel speed (Figure 11a). This is consistent with the results of Figure 6, where the structure with the highest pore frequency per unit length arises at a weld power of $800 \mathrm{~W}$ for the $1016 \mathrm{~mm} \times \mathrm{min}^{-1}$ speed weld series. While these results are consistent, SLF provides a more insightful detail of the pore structures present; for example, in the case of $800 \mathrm{~W}$ welds formed at $1016 \mathrm{~mm} \times$ $\mathrm{min}^{-1}$ with a $120 \mathrm{~mm}$ focus lens, the pore interfaces are separated by a distance that is 0.39 times the center-to-center distance between neighboring pores on average. Additionally, it is valuable to point out that the SLF is in the range of 0.4 to 0.6 for welds with a broad range of process parameters, which indicates that characteristic pore interspacing is approximately the same as the characteristic pore diameter in these cases. This suggests that for many weld cases, the characteristic pore interspacing can be approximated by the average pore diameter, which is generally easier to measure. However, high SLF values $(>0.6)$ are observed at the lowest power and the highest speed, indicating that pores may be spaced farther apart relative to their size at low delivered energy (Table 2 and Figure 11).

\section{Pore size variability}

The measures presented above are useful in quantifying the influence of specific weld parameters on resultant pore microstructures. These measures revealed various degree of variability within each weld. For example, ISDs are not sharply peaked, indicating some pore surfaces have high curvatures while others have low curvatures. Here, we examine the width of the distribution for pore sizes to better elucidate the variability in this measure. To this end, the coefficient of variation (c.v.) associated with equivalent pore radii is calculated. The coefficient of variation provides a quantitative measure of the variability of any population by taking the ratio of the standard deviation $(\sigma)$ to the average $(\mu)$, Equation 4.

$$
\text { c.v. }=\frac{\sigma}{\mu}=\frac{\sqrt{\frac{1}{N} \sum_{i=1}^{N}\left(x_{i}-\mu\right)^{2}}}{\mu}
$$


The coefficient of variation is useful as it allows for comparison across populations having very different means, varying populations and dissimilar distributions. For distributions having a standard deviation less than the average, a c.v. less than one is returned, indicating very low variability in the population. Alternatively, c.v. values greater than unity indicate notable amounts of variability where the value of c.v. indicates how many times greater the variability in the distribution is than the mean. To illustrate the trend is consistent across both focal lenses the c.v. values will be presented for both the $80 \mathrm{~mm}$ and $120 \mathrm{~mm}$ focal lens series, Figure 12. While no trend in pore size variability is apparent for changes in weld speed a minor increase in variability can be observed with an increase in delivered power. Additionally, the values of c.v. found for the pore size distributions, which are all well below unity, indicate that the spread in radii for all weld schedules are relatively small with respect to their mean value. Variability measures such as the one presented above will likely aid the understanding of microstructure's influence on mechanical response.

\section{Conclusions}

In this paper, quantitative characterization of porosity in laser-welds of 304L stainless steel has been performed non-destructively for 54 unique continuous-wave weld schedules via micro-computed tomography where each weld schedule represents a unique dataset. Direct correlations of pore size, shape, frequency, directionality, pore interspacing and solid linear fraction (SLF) with weld processing parameters have been made.

We find:

- Average and maximum pore volume increase with decreasing speed or increasing power.

- Pore frequency initially increases and then decreases with increasing power for a given travel speed.

- Interfacial shape distributions (ISDs) and interfacial normal distributions (INDs) illustrate that basic pore shape and directionality are similar for a given welding speed regardless of power delivered.

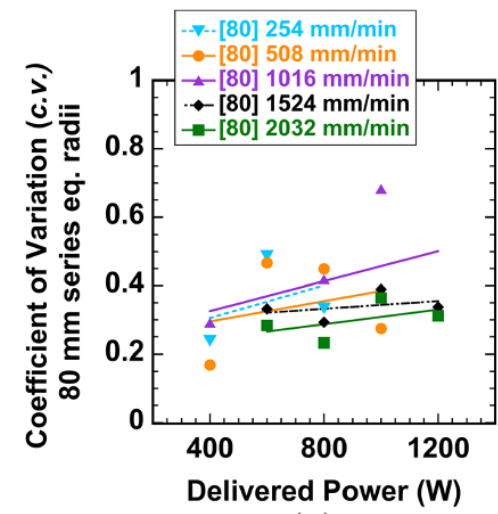

(a)

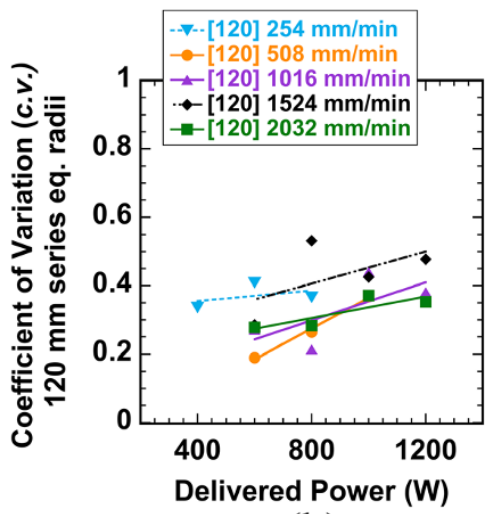

(b)

Figure 12 Coefficient of variation for equivalent pore radii for (a) $80 \mathrm{~mm}$ and (b) $120 \mathrm{~mm}$ focal distance welds indicating very small variability in pore size across all weld schedules examined. 
- ISDs show that pore shapes are nearly spherical or ellipsoidal at low and high travel speeds and are far more irregular, with a mix of ellipsoidal and saddle-shape geometries at moderate travel speeds.

- INDs indicate that pore orientations become anisotropic at moderate to high travel speeds with large concentrations of pore interfacial normals pointing toward and away from the direction of laser incidence.

- Characteristic pore interspacing is nominally equivalent to characteristic pore diameter for welds with a broad range of process parameters, as reflected in the solid linear fraction (SLF) values.

- The values of c.v. indicate that the spread in pore radii is small with respect to their mean value for all weld schedules.

- High travel speeds and low delivered power result in the lowest pore linear frequency while increasing the amount of solid material between pores, which would likely yield improved mechanical properties.

\section{Availability of supporting data}

Animations of the five primary 3D reconstructions featured in this article for which ISDs, INDs, pore interspacing and SLF were calculated and presented have been made publicly available [27].

\section{Additional files}

Additional file 1: 3D Reconstructions of Porosity produced by laser-weld under a focal lens of $80 \mathrm{~mm}$ at various speeds and powers.

Additional file 2: 3D Reconstructions of Porosity produced by laser-weld under a focal lens of $120 \mathrm{~mm}$ at various speeds and powers.

Additional file 3: Interfacial Shape Distributions for Porosity produced by laser-weld under a focal lens of $80 \mathrm{~mm}$ at various speeds and powers.

Additional file 4: Interfacial Shape Distributions for Porosity produced by laser-weld under a focal lens of $120 \mathrm{~mm}$ at various speeds and powers.

Additional file 5: Equal Area Interfacial Normal Distributions with respect to the positive $\mathrm{x}$ axis for Porosity produced by laser-weld under a focal lens of $80 \mathrm{~mm}$.

Additional file 6: Equal Area Interfacial Normal Distributions with respect to the negative $\mathrm{x}$ axis for Porosity produced by laser-weld under a focal lens of $80 \mathrm{~mm}$.

Additional file 7: Equal Area Interfacial Normal Distributions with respect to the positive $\mathrm{x}$ axis for Porosity produced by laser-weld under a focal lens of $120 \mathrm{~mm}$.

Additional file 8: Equal Area Interfacial Normal Distributions with respect to the negative $\mathrm{x}$ axis for Porosity produced by laser-weld under a focal lens of $120 \mathrm{~mm}$.

Competing interests

The authors declare that they have no competing interests.

Authors' contributions

JM coordinated data collection, performed image segmentation, created $3 d$ reconstructions and made basic characterization measures for all datasets. ISD and IND calculations were performed by LA and JM. Pore Interspacing and SLF calculations were performed by VC and KT. All authors read and approved the final manuscript.

\section{Acknowledgements}

Sandia National Laboratories is a multi-program laboratory managed and operated by Sandia Corporation, a wholly owned subsidiary of Lockheed Martin Corporation, for the US Department of Energy's National Nuclear Security Administration under contract DE-AC04-94AL85000. V.W.L. Chan and K. Thornton would like to acknowledge NSF DMR Grant \# 0746424 "CAREER: Integrated Research and Education Program in Three-Dimensional Materials Science and Visualization." The computational resources for calculations of pore interspacing and pore sizes were provided by the Extreme Science and Engineering Discovery Environment (XSEDE), which is supported by National Science Foundation grant number OCl-1053575, under allocation No. TG-DMR110007. 


\section{Author details}

${ }^{1}$ Computational Materials \& Data Science, Sandia National Laboratories, 87185 Albuquerque, NM, USA. ${ }^{2}$ Materials Science \& Engineering, University of Michigan, 48109 Ann Arbor, MI, USA.

Received: 11 November 2013 Accepted: 21 March 2014

Published: 29 April 2014

\section{References}

1. Mazumder J (1993) Laser-Beam Welding. In: ASM Handbook, Vol. 6. ASM International, Materials Park, OH, pp 262-269

2. Webber T, Lieb T, Mazumder J (2011) Laser Beam Welding. In: ASM Handbook, Vol. 6A. ASM International, Materials Park, OH, pp 556-569

3. Boyce BL, Reu PL, Robino CV (2006) The constitutive behavior of laser welds in 304L stainless steel determined by digital image correlation. Metall Mater Trans A 37A:2481-2492

4. Haboudou A, Peyre P, Vannes AB, Peix G (2003) Reduction of porosity content generated during Nd:YAG laser welding of A356 and AA5083 aluminum alloys. Mater Sci Eng A A363:40-52

5. Feist WD, Tillack G-R (1997) Ultrasonic Inspection of Pores in Electron Beam Welds. In: European-American Workshop Determination of Reliability and Validation Methods of NDE. BAM, Berlin, Germany. June 18-20 1997. vol 4. NDT.net

6. Zhu X, Shyam A, Jones JW, Mayer H, Lasecki JV, Allison JE (2006) Effects of microstructure and temperature on fatigue behavior of E319-T7 cast aluminum alloy in very long life cycles. Int J Fatigue 28:1566-1571

7. Shyam A, Picard YN, Jones JW, Allison JE, Yalisove SM (2004) Small fatigue crack propagation from micronotches in the cast aluminum alloy W319. Scr Mater 50:1109-1114. doi:10.1016/j.scriptamat.2004.01.031

8. Kuo TY, Jeng SL (2005) Porosity reduction in Nd-YAG laser welding of stainless steel and inconel alloy by using a pulsed wave. J Phys D Appl Phys 38:722-728. doi:10.1088/0022-3727/38/5/009

9. Madison J, Aagesen LK (2012) Porosity in Millimeter-Scale Welds of Stainless Steel: Three-Dimensional Characterization. Sandia National Laboratories, Albuquerque, NM

10. Madison J, Aagesen LK (2012) Quantitative characterization of porosity in laser welds of stainless steel. Scr Mater 67(9):783-786. doi:10.1016/j.scriptamat.2012.06.015

11. Tucker JD, Nolan TK, Martin AJ, Young GA (2012) Effect of travel speed and beam focus on porosity in alloy 690 laser welds. JOM 64(12):1409-1417. doi:10.1007/s11837-012-0481-3

12. Norris JT, Perricone MJ, Roach RA, Faraone KM, Ellison CM (2007) Evaluation of Weld Porosity in Laser Beam Seam Welds: Optimizing Continuous Wave and Square Wave Modulated Processes. Sandia National Laboratories, Albuquerque, NM

13. Norris JT, Robino CV, Hirschfeld DA, Perricone MJ (2011) Effects of laser parameters on porosity formation: investigating millimeter scale continuous wave Nd:YAG laser welds. Weld J 90:198-203

14. Madison JD, Aagesen LK, Battaile CC, Rodelas JM, Payton TKCS (2013) Coupling 3D quantitative interrogation of weld microstructure with 3D models of mechanical response. Metallography, Microstructure and Analysis 2 (6):359-363. doi:10.1007/s13632-013-0097-1

15. Matsunawa A, Kim J-D, Seto N, Mizutani M, Katayama S (1998) Dynamics of keyhole and molten pool in laser welding. J Laser Apps 10(6):247-254. doi:10.2351/1.521858

16. Pang S, Chen L, Zhou J, Yin Y, Chen T (2011) A three-dimensional sharp interface model for self-consistent keyhole and weld pool dynamics in deep penetration laser welding. J Phys D 44:1-15. do: 10.1088/0022-3727/44/2/025301

17. Rai R, Elmer JW, Palmer TA, DebRoy $T$ (2007) Heat transfer and fluid flow during keyhole mode laser welding of tantalum, Ti-6Al-4 V, 304L stainless steel and vanadium. J Phys D Appl Phys 40:5753-5766. doi:10.1088/0022-3727/40/18/037

18. Zhou J, Tsai H-L (2007) Porosity formation and prevention in pulsed laser welding. Trans ASME 129:1014-1024. doi:10.1115/1.2724846

19. Alkemper J, Voorhees PW (2001) Three-dimensional characterization of dendritic microstructures. Acta Mater 49:897-902

20. Mendoza R, Alkemper J, Voorhees PW (2003) The morphological evolution of dendritic microstructures during coarsening. Metall Mater Trans A 34A(3):481-489

21. Mendoza R, Savin I, Thornton K, Voorhees PW (2004) Topological complexity and the dynamics of coarsening. Nat Mater 3:385-388. doi:10.1038/nmat1138

22. Kammer D, Mendoza R, Voorhees PW (2006) Cylindrical domain formation in topologically complex structures. Scr Mater 55(1):17-22. doi:10.1016/j.scriptamat.2006.02.027

23. Kammer D, Voorhees PW (2006) The morphological evolution of dendritic microstructures during coarsening. Acta Mater 54(6):1549-1558. doi:10.1016/j.actamat.2005.11.031

24. Fife JL, Voorhees PW (2009) The morphological evolution of equiaxed dendritic microstructures during coarsening. Acta Mater 57:2418-2428. doi: 10.1016/j.actamat.2009.01.036

25. Meyer N, Desbrun M, Schroder P, Barr AH (2003) Discrete Differential-Geometry Operators for Triangulated 2-Manifolds. In: Polthier K, Hege HC (ed) Visualization and Mathematics III. Springer, Berlin, Germany, pp 35-37

26. Chan WWL, Thornton K (2012) Channel size distribution of complex three-dimensional microstructures calculated from the topological characterization of isodistance structures. Acta Mater 60:2509-2517. doi:10.1016/j.actamat.2011.12.042

27. Madison JD, Aagesen LK, Chan WWL, Thornton K (2014) 3-Dimensional Reconstructions of Porosity from Laser-Welds of 304L Stainless Steel at 600W and a Variety of Travel Speeds. http://hdl.handle.net/11115/243

doi:10.1186/2193-9772-3-11

Cite this article as: Madison et al:: Advancing quantitative description of porosity in autogenous laser-welds of 304L stainless steel. Integrating Materials and Manufacturing Innovation 2014 3:11. 